\title{
A Unified Treatment of Single Component Replacement Models
}

\author{
Hans Frenk, Rommert Dekker and Marcel Kleijn \\ Econometric Institute, Erasmus University Rotterdam, P.O. BOX 1738, 3000 DR ROTTERDAM, \\ The Netherlands
}

\begin{abstract}
In this paper we discuss a general framework for single component replacement models. This framework is based on the regenerative structure of these models and by using results from renewal theory a unified presentation of the discounted and average finite and infinite horizon cost models is given. Finally, some well-known replacement models are discussed, and making use of the previous results an easy derivation of their cost functions is presented.
\end{abstract}

Key Words: Maintenance, Renewal Theory, Cost Intensity Function.

\section{Introduction}

In the extensive literature on infinite horizon single component replacement models (for surveys, see McCall [17], Pierskalla and Voelker [21], Sherif and Smith [25] and Valdez-Flores and Feldman [27]) a lot of different models are analyzed. However, as observed by Zijlstra [30,31], in most of these models the replacement action, prescribed by a given stationary policy, regenerates the system, i.e. the system is "as good as new" immediately after the completion of such an action. Although this observation is always used in the literature, no paper discusses in detail the consequences of the regenerative structure of infinite horizon single component replacement models. In this paper we will start in Section 2 with such a regenerative structure. Using well-known techniques and results from the theory of regenerative processes, we will unify the derivation of the infinite horizon average and expected discounted costs associated with a given stationary policy. Moreover, we show that under some weak nonlattice assumption the difference between the expected discounted costs (with discount factor $\alpha>0$ ) up to time $t$ and the infinite horizon expected discounted costs, converges exponentially fast with factor $\alpha$ to an easy function of the corresponding infinite horizon average costs. Also in this section we derive easy upper and lower bounds on the cost functions of the corresponding finite horizon model. By the results of the first section and using a so-called cost intensity function it is now relatively easy to derive the cost function of a large 
class of replacement models with or without minimal repairs. This will be discussed in Section 3 and due to the absense of differentiability requirements for the preventive repair cost function and the regular maintenance cost function, these models are more general than those considered by Berg $[4,5,6]$. In fact, one cannot apply to these models the intuitively appealing Marginal Cost Analysis (MCA) method of Berg.

\section{General Setup}

In most single-component maintenance models we encounter the following framework. Let $c_{i}, i \geq 1$, be a sequence of independent and identically distributed random variables defined on a probability space $(\Omega, \mathscr{F}, \mathbb{P})$ with finite mean and (right continuous) distribution function $F$ satisfying $F(0)=0$ (Feller [11]). Moreover, set $c_{0}:=0$. The $n$th partial sum $S_{n}, n \geq 1$, of the random variables $c_{i}$, $i \geq 1$, denotes now the occurrence of the $n$th regeneration point associated with a given stationary replacement policy $p \in \mathscr{A}$. For notational convenience set also $S_{0}:=0$. Associated with this policy $p \in \mathscr{A}$ is an increasing nonnegative stochastic process $\left\{\boldsymbol{R}_{\alpha}(t), 0 \leq t<\infty\right\}$, defined on the same probability space $(\Omega, \mathscr{F}, \mathbb{P})$ with right continuous sample paths $\mathbb{P}$-a.s. $\left(\mathbb{P}\right.$-almost surely), $\boldsymbol{R}_{\alpha}(0)=0$ and $\boldsymbol{R}_{\alpha}(t, \cdot)$ for each $t>0$ a Borel measurable function. This process denotes the total discounted costs, with discount factor $\alpha \geq 0$, up to time $t$ due to policy $p \in \mathscr{A}$, and satisfies the following important regenerative property.

\section{Regeneration Property}

For each $n \geq 0$ the stochastic process

$$
\left\{\boldsymbol{c}_{n+1}, \boldsymbol{c}_{n+2}, \ldots,\left\{\exp \left(\alpha \boldsymbol{S}_{n}\right)\left(\boldsymbol{R}_{\alpha}\left(t+\boldsymbol{S}_{n}\right)-\boldsymbol{R}_{\alpha}\left(\boldsymbol{S}_{n}\right)\right): t \geq 0\right\}\right\}
$$

is independent of $\boldsymbol{c}_{1}, \ldots, \boldsymbol{c}_{n}$ and its distribution does not depend on $n$.

To relate the above setup with replacement models, we consider the following examples. For the age replacement family of single component maintenance models it follows that $c_{i}=T \wedge X_{i}$, with $T$ some positive parameter and $X_{i}$, $i \geq 1$, a sequence of independent and identically distributed random variables on $[0, \infty)$. Moreover, $T \wedge X_{i}:=\min \left\{T, X_{i}\right\}$. Also, for the block replacement family we obtain that $c_{i}=T$, with $T$ some positive parameter. Examples of generalizations of the latter policy are given by the opportunity-based block 
replacement policies (Dekker and Smeitink [10]) and the so-called T-policy which replaces the component at the first failure after reaching the age $T>0$ (Muth [19], Makis and Jardine [16]). If a component fails before reaching age $T$ it is assumed in most of the above models that it is minimally repaired. For both extensions of the block replacement rule it is clear that $c_{i}=T+\boldsymbol{Y}_{i}(T)$ with $Y_{i}(T), i \geq 1$, a sequence of independent and identically distributed nonnegative random variables depending on $T$. Finally, if one takes the random down time of the system into account in a block (Sivazlian [26]) or age replacement model, it is also easy to write down an explicit formula for $\boldsymbol{c}_{i}$. This shows some examples of replacement models fitting into the above framework. We will now continue with the analysis of this framework. In the remainder it is always assumed that the random variable $\boldsymbol{R}_{\alpha}\left(\boldsymbol{c}_{1}\right)$ is $\mathbb{P}$-integrable (page 37 of Ash [1]) or equivalently $\mathbb{E}\left(\boldsymbol{R}_{\alpha}\left(\boldsymbol{c}_{1}\right)\right)$ is finite. Since $\boldsymbol{R}_{\alpha}\left(\boldsymbol{c}_{1}\right) \leq \boldsymbol{R}_{0}\left(\boldsymbol{c}_{1}\right)$ for every $\alpha \geq 0$ it is sufficient to demand that $\mathbb{E}\left(\boldsymbol{R}_{0}\left(\boldsymbol{c}_{1}\right)\right)$ is finite. Since we need in Lemma 2.2 that $\mathbb{E}\left(\boldsymbol{R}_{\alpha}(t)\right)<\infty$ for every $t \geq 0$, we make the following observations. Although this line of reasoning is well-known within renewal theory it is listed here for completeness. Let $N(t):=\inf \left\{n \geq 0: S_{n}>t\right\}$ denote the renewal counting process associated with the random variables $\boldsymbol{c}_{i}, i \geq 0$. For this counting process it is easy to show that $\mathbb{E}(N(t))$ is finite for every $t \geq 0$ due to $F(0)=0<1$ (Asmussen [2]). Applying now the monotonicity of the stochastic process $\left\{\boldsymbol{R}_{\alpha}(t): 0 \leq\right.$ $t<\infty\}$ it follows for every $t \geq 0$ that

$$
0 \leq \boldsymbol{R}_{\alpha}(t) \leq \sum_{n=1}^{N(t)}\left(\boldsymbol{R}_{\alpha}\left(\boldsymbol{S}_{n}\right)-\boldsymbol{R}_{\alpha}\left(\boldsymbol{S}_{n-1}\right)\right) \leq \sum_{n=1}^{N(t)} \exp \left(\alpha \boldsymbol{S}_{n-1}\right)\left(\boldsymbol{R}_{\alpha}\left(\boldsymbol{S}_{n}\right)-\boldsymbol{R}_{\alpha}\left(\boldsymbol{S}_{n-1}\right)\right)
$$

By the regeneration property we obtain that the distribution of the random variable $\exp \left(\alpha \boldsymbol{S}_{n-1}\right)\left(\boldsymbol{R}_{\alpha}\left(\boldsymbol{S}_{n}\right)-\boldsymbol{R}_{\alpha}\left(\boldsymbol{S}_{n-1}\right)\right)$ is independent of $n$ and this yields that the expectation $\mathbb{E}\left(\exp \left(\alpha \boldsymbol{S}_{n-1}\right)\left(\boldsymbol{R}_{\alpha}\left(\boldsymbol{S}_{n}\right)-\boldsymbol{R}_{\alpha}\left(\boldsymbol{S}_{n-1}\right)\right)\right)=\mathbb{E}\left(\boldsymbol{R}_{\alpha}\left(\boldsymbol{c}_{1}\right)\right)<\infty$. Moreover, by the same regeneration property the random variable $\exp \left(\alpha \boldsymbol{S}_{n-1}\right)\left(\boldsymbol{R}_{\alpha}\left(\boldsymbol{S}_{n}\right)-\right.$ $\left.\boldsymbol{R}_{\alpha}\left(\boldsymbol{S}_{n-1}\right)\right)$ is independent of $\boldsymbol{c}_{1}, \ldots, \boldsymbol{c}_{n-1}$ and so it follows by Wald's identity (Wolff [29]) and $N(t)$ is a stopping time with respect to $c_{i}, i \geq 1$, satisfying $\mathbb{E}(N(t))<\infty$ for every $t>0$ that

$$
\begin{aligned}
0 & \leq \mathbb{E}\left(\boldsymbol{R}_{\alpha}(t)\right) \leq \mathbb{E}\left(\sum_{n=1}^{N(t)} \exp \left(\alpha \boldsymbol{S}_{n-1}\right)\left(\boldsymbol{R}_{\alpha}\left(\boldsymbol{S}_{n}\right)-\boldsymbol{R}_{\alpha}\left(\boldsymbol{S}_{n-1}\right)\right)\right) \\
& =\mathbb{E}(\boldsymbol{N}(t)) \mathbb{E}\left(\boldsymbol{R}_{\alpha}\left(\boldsymbol{c}_{1}\right)\right)<\infty
\end{aligned}
$$

To analyze the behaviour of this expectation as $t$ approaches infinity we introduce the related stochastic process $\left\{\boldsymbol{R}_{\alpha}\left(t \wedge \boldsymbol{c}_{1}\right): 0 \leq t<\infty\right\}$ and the function $f_{\alpha}:[0, \infty) \rightarrow[0, \infty]$ given by $f_{\alpha}(t):=\mathbb{E}\left(\boldsymbol{R}_{\alpha}\left(t \wedge \boldsymbol{c}_{1}\right)\right)$. Clearly this process represents the total discounted costs with discount factor $\alpha \geq 0$ made in the first cycle up to time $t$. It is now easy to establish the following result. 
Lemma 2.1: The function $f_{\alpha}:[0, \infty] \rightarrow[0, \infty)$ is uniformly bounded, nonnegative, increasing and right continuous with at most a countable number of discontinuities. Also it holds that $f_{\alpha}(\infty):=\lim _{t \rightarrow \infty} f_{\alpha}(t)=\mathbb{E}\left(\boldsymbol{R}_{\alpha}\left(\boldsymbol{c}_{1}\right)\right)<\infty$.

Proof: Since the stochastic process $\left\{\boldsymbol{R}_{\alpha}(t): 0 \leq t<\infty\right\}$ is increasing and nonnegative it follows that the function $f_{\alpha}$ is nonnegative and increasing. Moreover, since $\left\{\boldsymbol{R}_{\alpha}(t): 0 \leq t<\infty\right\}$ has right continuous sample paths $\mathbb{P}$-a.s. we observe that $\lim _{h \downarrow_{0}} \boldsymbol{R}_{\alpha}\left((t+h) \wedge \boldsymbol{c}_{1}\right)=\boldsymbol{R}_{\alpha}\left(t \wedge \boldsymbol{c}_{1}\right) \mathbb{P}$-a.s. and due to $\boldsymbol{c}_{1}<\infty \mathbb{P}$-a.s. also $\lim _{t \uparrow \infty} \boldsymbol{R}_{\alpha}\left(t \wedge c_{1}\right)=\boldsymbol{R}_{\alpha}\left(c_{1}\right) \mathbb{P}$-a.s. Applying now the dominated convergence theorem (Ash [1]) and $\mathbb{E}\left(\boldsymbol{R}_{\alpha}\left(\boldsymbol{c}_{1}\right)\right)<\infty$ this yields that $f_{\alpha}$ is right continuous and $f_{\alpha}(\infty)=\mathbb{E}\left(\boldsymbol{R}_{\alpha}\left(\boldsymbol{c}_{1}\right)\right)$. Finally, due to $f_{\alpha}$ increasing and finite, we obtain by Theorem 4.1.2 of Mikolás [18] that $f_{\alpha}$ has a set of discontinuities which is at most countable.

It is also easy to relate the discounted stochastic process $\left\{\boldsymbol{R}_{\alpha}\left(t \wedge \boldsymbol{c}_{1}\right): 0 \leq\right.$ $t<\infty\}$ to its undiscounted version $\left\{\boldsymbol{R}_{0}\left(t \wedge c_{1}\right): 0 \leq t<\infty\right\}$. Since the stochastic process $\left\{\boldsymbol{R}_{\alpha}\left(t \wedge \boldsymbol{c}_{1}\right): 0 \leq t<\infty\right\}$ is increasing and $\boldsymbol{R}_{0}\left(\boldsymbol{c}_{1}\right)<\infty$ P-a.s. it follows by Theorem 5.7.3 of Mikolás [18] that for almost every realization $\omega \in \Omega$ the value $\boldsymbol{R}_{\alpha}\left(\left(t \wedge \boldsymbol{c}_{1}\right)(\omega), \omega\right)$ can be written as the Riemann-Stieltjes integral

$$
\int_{0}^{\left(t \wedge c_{1}\right)(\omega)} \exp (-\alpha y) \boldsymbol{R}_{0}(d y, \omega)
$$

This implies that

$$
f_{\alpha}(t):=\mathbb{E}\left(\boldsymbol{R}_{\alpha}\left(t \wedge c_{1}\right)\right)=\mathbb{E}\left(\int_{0}^{t \wedge c_{1}} \exp (-\alpha y) \boldsymbol{R}_{0}(d y)\right)
$$

and by the extended monotone convergence theorem (Ash [1]) and Lemma 2.1 it follows that

$$
\mathbb{E}\left(\boldsymbol{R}_{\alpha}\left(\boldsymbol{c}_{1}\right)\right)=\mathbb{E}\left(\int_{0}^{\boldsymbol{c}_{1}} \exp (-\alpha y) \boldsymbol{R}_{0}(d y)\right)
$$

To unify the derivation of the finite-valued function $v_{\alpha}:[0, \infty) \rightarrow[0, \infty)$ defined by $\left.v_{\alpha}(t):=\mathbb{E}\left(\boldsymbol{R}_{\alpha}(t)\right)\right)$ we show in the next lemma that for every discount factor $\alpha \geq 0$ it is the unique solution of a renewal type equation. However, before presenting this lemma we introduce for $\alpha \geq 0$ the (possibly) defective distribution function $F_{\alpha}$ given by 


$$
F_{\alpha}(x):=\int_{0}^{x} \exp (-\alpha y) F(d y)
$$

Moreover, we introduce the renewal function $U_{\alpha}:[0, \infty) \rightarrow[0, \infty)$ associated with $F_{\alpha}$ and this function has the well-known representation

$$
U_{\alpha}(x):=\sum_{n=0}^{\infty} F_{\alpha}^{n *}(x)
$$

where $F_{\alpha}^{n *}$ denotes the $n$-fold convolution of $F_{\alpha}$.

Observe now by Lemma 2.1 and $U_{\alpha}$ is right continuous due to $F_{\alpha}$ is right continuous that by Exercise 2 at page 56 of Ash [1] the integral in the next lemma can be interpreted as a Riemann-Stieltjes integral.

Lemma 2.2: It follows for every discount factor $\alpha \geq 0$ that

$$
v_{\alpha}(t)=\int_{0^{-}}^{t} f_{\alpha}(t-y) U_{\alpha}(d y)
$$

for every $t \geq 0$.

Proof: Clearly for every $t \geq 0$ we obtain that

$$
v_{\alpha}(t)=f_{\alpha}(t)+\int_{0}^{t} \mathbb{E}\left(\boldsymbol{R}_{\alpha}(t)-\boldsymbol{R}_{\alpha}\left(t \wedge \boldsymbol{c}_{1}\right) \mid \boldsymbol{c}_{1}=y\right) F(d y)
$$

By the regeneration property of the stochastic process $\left\{\boldsymbol{R}_{\alpha}(t): 0 \leq t<\infty\right\}$ it follows for every $y \leq t$ that

$$
\begin{aligned}
\mathbb{E}\left(\boldsymbol{R}_{\alpha}(t)-\boldsymbol{R}_{\alpha}\left(t \wedge c_{1}\right) \mid \boldsymbol{c}_{1}=y\right) \\
\quad=\mathbb{E}\left(\boldsymbol{R}_{\alpha}\left(t-y+\boldsymbol{c}_{1}\right)-\boldsymbol{R}_{\alpha}\left(\boldsymbol{c}_{1}\right) \mid \boldsymbol{c}_{1}=y\right) \\
=\exp (-\alpha y) \mathbb{E}\left(\exp \left(\alpha \boldsymbol{c}_{1}\right)\left(\boldsymbol{R}_{\alpha}\left(t-y+\boldsymbol{c}_{1}\right)-\boldsymbol{R}_{\alpha}\left(\boldsymbol{c}_{1}\right)\right) \mid \boldsymbol{c}_{1}=y\right) \\
=\exp (-\alpha y) \mathbb{E}\left(\exp \left(\alpha c_{1}\right)\left(\boldsymbol{R}_{\alpha}\left(t-y+\boldsymbol{c}_{1}\right)-\boldsymbol{R}_{c}\left(\boldsymbol{c}_{1}\right)\right)\right) \\
=\exp (-\alpha y) \mathbb{E}\left(\boldsymbol{R}_{\alpha}(t-y)\right)
\end{aligned}
$$


and this implies

$$
v_{\alpha}(t)=f_{\alpha}(t)+\int_{0}^{t} \exp (-\alpha y) v_{\alpha}(t-y) F(d y)=f_{\alpha}(t)+\int_{0}^{t} v_{\alpha}(t-y) F_{\alpha}(d y)
$$

By Lemma 2.1 and $\mathbb{E}\left(\boldsymbol{R}_{\alpha}(t)\right)$ is bounded on every compact interval the desired result follows by applying Theorem 2.4 in Chapter 4 of Asmussen [2].

By Lemma 2.1 it follows immediately that the function $G_{\alpha}:[0, \infty) \rightarrow[0,1]$ given by $G_{\alpha}(t):=f_{\alpha}(t) / f_{\alpha}(\infty)$ is a right continuous nondefective distribution function satisfying $G_{a}(0)=0$. Hence, by Lemma 2.1 and Lemma 2.2 we obtain that

$$
v_{\alpha}(t)=f_{\alpha}(\infty) U_{\alpha}^{d}(t)=\mathbb{E}\left(\boldsymbol{R}_{\alpha}\left(c_{1}\right)\right) U_{\alpha}^{d}(t)
$$

with $U_{\alpha}^{d}(t):=\int_{0^{-}}^{t} G_{\alpha}(t-y) U_{\alpha}(d y)$ the renewal function associated with the delayed renewal process $\left\{\boldsymbol{S}_{n}^{d}: n \geq 0\right\}$ where $\boldsymbol{S}_{n}^{d}=\boldsymbol{S}_{n}+\boldsymbol{d}_{\alpha}$ and $\boldsymbol{d}_{\alpha}$ a random variable with distribution function $G_{\alpha}$. By this observation one can easily verify for $\alpha=0$ the following result.

Theorem 2.1: It follows that the infinite horizon average costs associated with a given stationary policy $p \in \mathscr{A}$ equal

$$
c_{0}(p):=\lim _{t \rightarrow \infty} \frac{v_{0}(t)}{t}=\frac{\mathbb{E}\left(\boldsymbol{R}_{0}\left(\boldsymbol{c}_{1}\right)\right)}{\mathbb{E}\left(\boldsymbol{c}_{1}\right)}
$$

If additionally the distribution function $F$ is nonlattice and $\mathbb{E}\left(c_{1}^{2}\right)$ is finite, we obtain that

$$
\lim _{t \rightarrow \infty}\left(v_{0}(t)-\frac{1}{\mathbb{E}\left(\boldsymbol{c}_{1}\right)} \int_{0}^{t} \mathbb{E}\left(\boldsymbol{R}_{0}\left(y \wedge c_{1}\right)\right) d y\right)=\frac{\mathbb{E}\left(\boldsymbol{c}_{1}^{2}\right)}{2 \mathbb{E}\left(\boldsymbol{c}_{1}\right)} c_{0}(p)
$$

Proof: By the elementary renewal theorem (see Proposition 1.4 of Chapter 4 in Asmussen [2]) we obtain that $\lim _{t \rightarrow \infty} U_{0}^{d}(t) / t=1 / \mathbb{E}\left(c_{1}\right)$ and this shows by (2) the first part. Moreover, if $F$ is nonlattice and $\mathbb{E}\left(c_{1}^{2}\right)$ is finite we obtain by Proposition 4.1 of Chapter 6 in Asmussen [2] that

$$
\lim _{t \rightarrow \infty}\left(U_{0}(t)-\frac{t}{\mathbb{E}\left(c_{1}\right)}\right)=\frac{\mathbb{E}\left(c_{1}^{2}\right)}{2\left(\mathbb{E}\left(c_{1}\right)\right)^{2}}
$$


Hence, it follows that

$$
\begin{aligned}
\lim _{t \rightarrow \infty}\left(U_{0}^{d}(t)-\frac{1}{\mathbb{E}\left(c_{1}\right)} \int_{0}^{t} G_{0}(y) d y\right) & =\lim _{t \rightarrow \infty} \int_{0}^{t}\left(U_{0}(t-y)-\frac{(t-y)}{\mathbb{E}\left(c_{1}\right)}\right) G_{0}(d y) \\
& =\frac{\mathbb{E}\left(c_{1}^{2}\right)}{2\left(\mathbb{E}\left(c_{1}\right)\right)^{2}}
\end{aligned}
$$

and this shows by the first part, relation (2) and the definition of $G_{0}$ the desired result.

As already noticed the random variable $c_{i}$ associated with the age replacement family of single component maintenance models has the representation $\boldsymbol{c}_{i}=T \wedge X_{i}$ and so we immediately obtain that $\mathbb{E}\left(c_{i}^{2}\right)$ is finite. If additionally the distribution function $F$ of the random variable $c_{i}=T \wedge X_{i}$ is nonlattice, it follows that the conditions for the second part of Theorem 2.1 are satisfied. Observe in Section 4 we will write out the formulas of Theorem 2.1 for a large class of age replacement cost models. Similar remarks can be made for the class of random variables $\boldsymbol{c}_{i}=T+\boldsymbol{Y}_{i}(T)$. For this class we need to assume that $\mathbb{E}\left(\left(\boldsymbol{Y}_{i}(T)\right)^{2}\right)$ is finite and the distribution function of the random variable $\boldsymbol{Y}_{i}(T)$ is nonlattice. Finally, for the block replacement family of single component maintenance models it follows due to $\boldsymbol{c}_{i}=T$ that the corresponding degenerate distribution function is clearly lattice. This means that one cannot apply the second part of Theorem 2.1. However, it is easy to derive a similar type of result by using Lemma 2.2. This is possible since the renewal function is an easy stepfunction with unit steps at multiples of $T$ if the underlying distribution function is degenerate at $T$.

Without any conditions on the distribution function $F$ except finiteness of the second moment $\mathbb{E}\left(\boldsymbol{c}_{1}^{2}\right)$ it is relatively easy to derive upper and lower bounds on the function $v_{0}$. This is a consequence of the existence of upper and lower bounds for the renewal function $U_{0}$ and therefore we will first discuss these bounds. If $c_{*}$ is a random variable independent of the $n$th partial sum $S_{n}, n \geq 1$, with distribution function $F_{e}$ given by

$$
F_{e}(x)=\frac{1}{\mathbb{E}\left(c_{1}\right)} \int_{0}^{x}(1-F(y)) d y
$$

then we consider the delayed renewal process $\left\{\boldsymbol{S}_{n}^{d}: n \geq 0\right\}$ with $\boldsymbol{S}_{n}^{d}=\boldsymbol{S}_{n}+\boldsymbol{c}_{*}$, $n \geq 0$. The distribution function $F_{e}$ is called the equilibrium distribution associated with $F$ and it is well-known (Asmussen [2]) that the renewal function $U_{e}$ of this delayed renewal process satisfies

$$
U_{e}(x)=\mathbb{E}\left(\sum_{n=0}^{\infty} 1_{\left\{\boldsymbol{s}_{n}^{d} \leq x\right\}}\right)=\mathbb{E}\left(U_{0}\left(x-\boldsymbol{c}_{*}\right) 1_{\left\{\boldsymbol{c}_{*} \leq x\right\}}\right)=\frac{x}{\mathbb{E}\left(\boldsymbol{c}_{1}\right)}
$$


By (3) and $U_{0}$ nonnegative and increasing we immediately obtain the wellknown linear lower bound $x / \mathbb{E}\left(c_{1}\right)=\mathbb{E}\left(U_{0}\left(x-c_{*}\right) 1_{\left\{c_{*} \leq x\right\}}\right) \leq U_{0}(x)$. Although it is relatively easy to improve this lower bound around zero (Van Weeren [28]) we will not pursue this. To construct an upper bound we observe the following. If $c^{*}$ is a random variable on $[0, \infty)$ independent of the random variables $c_{*}$ and $S_{n}, n \geq 0$, it follows by (3) that

$$
\begin{aligned}
\mathbb{E}\left(U_{0}\left(x+c^{*}-c_{*}\right)\right) & =\mathbb{E}\left(\mathbb{E}\left(U_{0}\left(x+c^{*}-c_{*}\right) 1_{\left\{c_{*} \leq x+c^{*}\right\}} \mid c^{*}\right)\right) \\
& =\frac{1}{\mathbb{E}\left(c_{1}\right)} \mathbb{E}\left(x+c^{*}\right)
\end{aligned}
$$

It is now possible to prove the following upper bound. This upper bound is known as Lorden's inequality and for completeness we list an easier proof as the one given in Proposition 4.2 in Chapter 6 of Asmussen [2].

Lemma 2.3: If $\mathbb{E}\left(c_{1}^{2}\right)$ is finite then for every $x \geq 0$ it follows that

$$
U_{0}(x) \leq \frac{x}{\mathbb{E}\left(c_{1}\right)}+\frac{\mathbb{E}\left(c_{1}^{2}\right)}{\left(\mathbb{E}\left(c_{1}\right)\right)^{2}}
$$

Proof: Let $c^{*}$ and $c_{*}$ be independent and identically distributed nonnegative random variables with distribution function $F_{e}$ and assume that these random variables are independent of the random variables $S_{n}, n \geq 0$. Using the subadditivity of the renewal function $U_{0}$, i.e. $U_{0}(x+y) \leq U_{0}(x)+U_{0}(y)$ for every $x$, $y \geq 0$ we obtain that

$$
U_{0}(x) \leq \mathbb{E}\left(U_{0}\left(x+c^{*}-c_{*}\right)\right)+\mathbb{E}\left(U_{0}\left(c_{*}-c^{*}\right)\right)
$$

and this implies by (4) that $U_{0}(x) \leq \frac{\mathbb{E}\left(x+c^{*}\right)}{\mathbb{E}\left(c_{1}\right)}+\frac{\mathbb{E}\left(c_{*}\right)}{\mathbb{E}\left(c_{1}\right)}$. Since $\mathbb{E}\left(c^{*}\right)=\mathbb{E}\left(c_{*}\right)=\frac{\mathbb{E}\left(c_{1}^{2}\right)}{2 \mathbb{E}\left(c_{1}\right)}$ the desired result follows.

It is now possible to derive the following upper and lower bound for the average cost up to time $t$ if the second moment $\mathbb{E}\left(c_{1}^{2}\right)$ is finite. This will be discussed in the next theorem, and the listed inequality should be compared with the second part of Theorem 2.1 . 
Theorem 2.2: If $\mathbb{E}\left(c_{1}^{2}\right)$ is finite then it follows for every $t>0$ that

$$
0 \leq v_{0}(t)-\frac{1}{\mathbb{E}\left(c_{1}\right)} \int_{0}^{t} \mathbb{E}\left(\boldsymbol{R}_{0}\left(y \wedge \boldsymbol{c}_{1}\right)\right) d y \leq \frac{\mathbb{E}\left(\boldsymbol{c}_{1}^{2}\right)}{\mathbb{E}\left(\boldsymbol{c}_{1}\right)} c_{0}(p)
$$

Proof: By Lemma 2.2 we obtain since $f_{0}(0)=0$ that

$$
v_{0}(t)=\int_{0^{-}}^{t} f_{0}(t-y) U_{0}(d y)=\int_{0^{+}}^{t} U_{0}(t-y) f_{0}(d y)
$$

Due to $f_{0}$ increasing this yields the desired inequality by substituting the lower and upper bound for the renewal function, given by Lemma 2.3, into the above expression.

By Theorem 2.2 it is clear for $t$ large enough and $\mathbb{E}\left(c_{1}^{2}\right)$ finite that a reasonable approximation for the average costs up to time $t$ is given by $\frac{1}{\mathbb{E}\left(\boldsymbol{c}_{1}\right)} \int_{0}^{t} \mathbb{E}\left(\boldsymbol{R}_{0}(y \wedge\right.$ $\left.\left.c_{1}\right)\right) d y$. This observation concludes our discussion of the case $\alpha=0$. We will now analyze the behaviour of the objective function for the discounted case. To start with this analysis we will first analyze the behaviour of the renewal function $U_{\alpha}$, introduced in (1).

Lemma 2.4: If $\alpha>0$ it follows for every $t>0$ that

$$
\frac{-1}{1-F_{\alpha}(\infty)} \leq \exp (\alpha t)\left(U_{\alpha}(t)-\frac{1}{1-F_{\alpha}(\infty)}\right) \leq 0
$$

Moreover, if $F$ is nonlattice we obtain that

$$
\lim _{t \rightarrow \infty} \exp (\alpha t)\left(U_{\alpha}(t)-\frac{1}{1-F_{\alpha}(\infty)}\right)=\frac{-1}{\alpha \mathbb{E}\left(c_{1}\right)}
$$

Proof: To prove the second part we observe since $F$ is nonlattice with finite mean that by (iv) of Proposition 4.1 in Chapter 4 of Asmussen [2] and the inequality

$$
\begin{aligned}
\exp (\alpha t)\left(F_{\alpha}(\infty)-F_{\alpha}(t)\right) & =\exp (\alpha t) \int_{t}^{\infty} \exp (-\alpha y) F(d y) \\
& \leq 1-F(t)
\end{aligned}
$$


the function $t \rightarrow \exp (\alpha t)\left(F_{\alpha}(\infty)-F_{\alpha}(t)\right)$ is directly Riemann integrable. Hence, by proposition 5.6 in Chapter 6 of Asmussen [2] (substitute $F_{\alpha}(t)$ for $z(t)$ ) the second part follows. To verify the first part it is shown in the proof of Proposition 5.6 in Chapter 6 of Asmussen [2] that

$$
U_{\alpha}(t)-\frac{1}{1-F_{\alpha}(\infty)}=\left(U_{\alpha} * z_{1}\right)(t)
$$

with $z_{1}(t):=\left(F_{\alpha}(t)-F_{\alpha}(\infty)\right) /\left(1-F_{\alpha}(\infty)\right)$. This implies that

$$
\exp (\alpha t)\left(U_{\alpha}(t)-\frac{1}{1-F_{\alpha}(\infty)}\right)=\int_{0^{-}}^{t} \exp (\alpha(t-y)) z_{1}(t-y) U_{0}(d y)
$$

and since $-\exp (\alpha t) z_{1}(t) \leq(1-F(t)) /\left(1-F_{\alpha}(\infty)\right)$ for every $t \geq 0$ we obtain that

$$
\begin{aligned}
0 & \geq \exp (\alpha t)\left(U_{\alpha}(t)-\frac{1}{1-F_{\alpha}(\infty)}\right) \\
& \geq \frac{-1}{1-F_{\alpha}(\infty)} \int_{0^{-}}^{t}(1-F(t-y)) U_{0}(d y) \\
& =\frac{-1}{1-F_{\alpha}(\infty)}
\end{aligned}
$$

An easy application of Lemma 2.4 is now given by the following result.

Theorem 2.3: It follows for $\alpha>0$ that

$$
\begin{aligned}
\lim _{t \rightarrow \infty} v_{\alpha}(t) & =\frac{\mathbb{E}\left(\int_{0}^{\boldsymbol{c}_{1}} \exp (-\alpha y) \boldsymbol{R}_{0}(d y)\right)}{1-F_{\alpha}(\infty)} \\
& =\frac{\mathbb{E}\left(\int_{0}^{c_{1}} \exp (-\alpha y) \boldsymbol{R}_{0}(d y)\right)}{\alpha \int_{0}^{\infty}(1-F(y)) \exp (-\alpha y) d y}
\end{aligned}
$$

Moreover, for every $t>0$ the inequality

$$
-c_{0}(p) \frac{\mathbb{E}\left(\boldsymbol{c}_{1}\right)}{1-F_{\alpha}(\infty)} \leq \exp (\alpha t)\left(v_{\alpha}(t)-v_{\alpha}(\infty)\right) \leq 0
$$


holds with $c_{0}(p)$ the value of the corresponding infinite horizon average costs. Finally, if additionaly the distribution function $F$ is nonlattice we obtain that

$$
\lim _{t \rightarrow \infty} \exp (\alpha t)\left(v_{\alpha}(t)-v_{\alpha}(\infty)\right)=\frac{-c_{0}(p)}{\alpha}
$$

Proof: By (2) it follows that

$$
\begin{aligned}
\lim _{t \rightarrow \infty} v_{\alpha}(t) & =f_{\alpha}(\infty) \lim _{t \rightarrow \infty} U_{\alpha}^{d}(t) \\
& =f_{\alpha}(\infty) \lim _{t \rightarrow \infty} \int_{0}^{t} U_{\alpha}(t-y) G_{\alpha}(d y) \\
& =f_{\alpha}(\infty) U_{\alpha}(\infty) \\
& =\frac{f_{\alpha}(\infty)}{1-F_{\alpha}(\infty)}
\end{aligned}
$$

As observed before Lemma 2.2 we know that $f_{\alpha}(\infty)=\mathbb{E}\left(\boldsymbol{R}_{\alpha}\left(\boldsymbol{c}_{1}\right)\right)=$ $\mathbb{E}\left(\int_{0}^{\boldsymbol{c}_{1}} \exp (-\alpha y) \boldsymbol{R}_{0}(d y)\right)$ and this shows the first part. To prove the remaining results we first observe by Lemma 2.1 and Theorem 5.7.3 of Mikolás [18] that $f_{\alpha}(t)=\int_{0}^{t} \exp (-\alpha y) f_{0}(d y)$ for every $0 \leq t<\infty$. This implies using $v_{\alpha}(\infty)=$ $f_{\alpha}(\infty) U_{\alpha}(\infty)$ and Lemma 2.2 that

$$
\begin{aligned}
\exp (\alpha t)\left(v_{\alpha}(t)-v_{\alpha}(\infty)\right)= & \int_{0^{-}}^{t} \exp (\alpha(t-y))\left(U_{\alpha}(t-y)-U_{\alpha}(\infty)\right) f_{0}(d y) \\
& +U_{\alpha}(\infty) \exp (\alpha t)\left(f_{\alpha}(t)-f_{\alpha}(\infty)\right)
\end{aligned}
$$

Since $0 \leq \exp (\alpha t)\left(f_{\alpha}(\infty)-f_{\alpha}(t)\right) \leq f_{0}(\infty)-f_{0}(t)$ the third part follows by the second part of Lemma 2.4. Moreover, by the above inequality, Theorem 2.1 and the first part of Lemma 2.4 we obtain for every $t \geq 0$ that

$$
\begin{aligned}
0 & \geq \exp (\alpha t)\left(v_{\alpha}(t)-v_{\alpha}(\infty)\right) \\
& \geq \frac{-f_{0}(t)}{1-F_{\alpha}(\infty)}+U_{\alpha}(\infty)\left(f_{0}(t)-f_{0}(\infty)\right) \\
& =\frac{-f_{0}(\infty)}{1-F_{\alpha}(\infty)}=-c_{0}(p) \frac{\mathbb{E}\left(c_{1}\right)}{1-F_{\alpha}(\infty)}
\end{aligned}
$$

and this shows the second part. 
Clearly the above lemma can be used to justify the approximation $v_{\alpha}(\infty)$ of the discounted cost $v_{x}(t)$ up to time $t$ for $t$ large enough. To be more precise, it follows by Theorem 2.3 that

$$
0 \leq v_{\alpha}(\infty)-v_{\alpha}(t) \leq \exp (-\alpha t) c_{0}(p) \frac{\mathbb{E}\left(c_{1}\right)}{1-F_{\alpha}(\infty)}
$$

In the next section we will consider some examples of replacement models fitting into the previous setup. At the same time we will show an easy and intuitively clear way to compute the corresponding objective function.

\section{Replacement Models and their Objective Functions}

Before considering some examples of replacement models fitting into the general setup, and computing by an intuitively appealing procedure their objective function, we need to derive a clear interpretation of the function $f_{\alpha}(t):=$ $\mathbb{E}\left(\boldsymbol{R}_{\alpha}\left(t \wedge c_{1}\right)\right)$. Observe by the Lebesgue decomposition theorem (Feller [11], Loeve [14]) that the function $f_{\alpha}$ might contain an increasing continuous singular component and this implies that such a clear interpretation probably does not exist. Fortunately it is possible for most of the replacement models to decompose (not necessarily unique!) the stochastic process $\left\{\boldsymbol{R}_{\alpha}\left(t \wedge c_{1}\right): 0 \leq\right.$ $t<\infty\}$ into an increasing stochastic process $\left\{\boldsymbol{Z}_{\alpha}(t): 0 \leq t<\infty\right\}$ with right continuous sample paths $\mathbb{P}$-a.s., $Z_{\alpha}(0)=0$ and $t \rightarrow \mathbb{E}\left(Z_{\alpha}(t)\right)$ continuous, and a stochastic process $\left\{g_{\alpha}\left(t \wedge c_{1}\right): 0 \leq t<\infty\right\}$ with $g_{\alpha}$ an increasing right continuous finite-valued function satisfying $g_{\alpha}(0)=0$. If we denote by $\left\{\boldsymbol{Z}_{0}(t): 0 \leq t<\right.$ $\infty\}$ the undiscounted version of the stochastic process $\left\{Z_{\alpha}(t): 0 \leq t<\infty\right\}$ then we introduce for this undiscounted version the so-called cost intensity function $m_{0}:[0, \infty) \rightarrow[-\infty,+\infty]$ given by

$$
m_{0}(t):=\limsup _{h \downarrow 0} \frac{\mathbb{E}\left(Z_{0}(t+h)-Z_{0}(t) \mid c_{1}>t\right)}{h}
$$

Moreover, if $g_{0}(t):=\int_{0}^{t} \exp (\alpha y) g_{\alpha}(d y)$ (the undiscounted version of $\left.g_{\alpha}\right)$ then it is possible to prove the following result (Frenk, Dekker and Kleijn [13]).

Theorem 3.1: If the above decomposition with the required properties holds, and the cost intensity function $m_{0}$ is finite-valued then it follows that 


$$
\begin{aligned}
\mathbb{E}\left(\boldsymbol{R}_{\alpha}\left(t \wedge \boldsymbol{c}_{1}\right)\right)= & \int_{0}^{t} \exp (-\alpha y) m_{0}(y)(1-F(y)) d y \\
& +\int_{0}^{t} \exp (-\alpha y)(1-F(y)) g_{0}(d y)
\end{aligned}
$$

for every $t \geq 0$.

The above result together with the results of the previous section yield an intuitively appealing approach to derive the objective function of a replacement model satisfying the above decomposition property. To show this we will consider in some detail a few replacement models. Observe this section considers similar models, with a more general cost structure, as considered in an intuitive way by Berg $[4,5,6]$. The first model we will consider is the age replacement model with running costs and age-dependent replacement costs. In this model a component is replaced by a new component either at failure (failure replacement) or preventively (preventive replacement) if it reaches a critical age $0<$ $T<\infty$. In this model the regeneration points are given by the replacement instances and so $c_{i}=T \wedge \boldsymbol{X}_{i}$ with $\boldsymbol{X}_{i}, i \geq 1$, a sequence of independent and identically distributed random variables with distribution function $G$ representing the random lifetime of the $i$ th component. We assume for convenience that the distribution function $G$ has a finite-valued density $g$. Moreover, the replacement costs both at a preventive and a failure replacement depend on the age of the component and are given by the increasing, finite-valued and right continuous functions $c_{p}(\cdot)$, resp. $c_{f}(\cdot)$. This means if a component of age $x<T$ breaks down, the replacement costs are given by $c_{f}(x)$, while at a preventive replacement of a component of age $T$ the corresponding costs are $c_{p}(T)$. Finally, it is assumed that the running costs of a component are given by the increasing, finite-valued and right continuous function $K:[0, \infty) \rightarrow[0, \infty)$ with $K(0)=0$ and these costs represent for example regular maintenance costs, depreciation costs, costs of reduced output and so on. For the above model the function $g_{0}$ is given by

$$
g_{0}(x)= \begin{cases}K(x) & \text { if } x<T \\ c_{p}(T)+K(T) & \text { if } x \geq T>0\end{cases}
$$

while the (right continuous) stochastic process $Z_{0}(t)$ is defined by

$$
Z_{0}(t)=\text { cost in the first cycle up to time } t \text { due to a replacement at failure }
$$

Observe now for $t \geq T>0$ that $Z_{0}(t-)=Z_{0}(t)$ and for $t<T$ that $Z_{0}(t-)<$ $Z_{0}(t)$ if and only if the first failure occurs at time $t$. By the continuity of the 
distribution function $G$ this implies that $Z_{0}(t-)=Z_{0}(t) \mathbb{P}$-a.s. and hence by a standard application of the dominated convergence theorem (Ash [1]) we obtain that $t \rightarrow \mathbb{E}\left(Z_{0}(t)\right)$ is a continuous function. To determine the cost intensity function notice for $t+h<T$ and $h>0$ that $Z_{0}(t+h)=Z_{0}(t)=0$ conditional on the event $\left\{c_{1}>t+h\right\}$ and so

$$
\mathbb{E}\left(Z_{0}(t+h)-Z_{0}(t) \mid c_{1}>t\right)=\mathbb{E}\left(\left(Z_{0}(t+h)-Z_{0}(t)\right) 1_{\left\{c_{1} \leq t+h\right\}} \mid c_{1}>t\right)
$$

This implies by the monotonicity of the function $c_{f}$ and the representation of $c_{1}$ that

$$
\begin{aligned}
c_{f}(t) \frac{G(t+h)-G(t)}{1-G(t)} & \leq \mathbb{E}\left(Z_{0}(t+h)-Z_{0}(t) \mid c_{1}>t\right) \\
& \leq c_{f}(t+h) \frac{G(t+h)-G(t)}{1-G(t)}
\end{aligned}
$$

for every $t+h<T$ and $h>0$. Since $c_{f}(\cdot)$ is a right continuous function and $G$ has a failure rate $r(\cdot)$ given by $r(t)=\frac{g(t)}{1-G(t)}$ for every $t \geq 0$ we finally obtain for $t<T$ that

$$
m_{0}(t):=\limsup _{h \downarrow 0} \frac{\mathbb{E}\left(Z_{0}(t+h)-Z_{0}(t) \mid c_{1}>t\right)}{h}=c_{f}(t) r(t)
$$

Moreover, for $t \geq T$ and $h>0$ it follows immediately that $Z_{0}(t+h)=Z_{0}(t)$ and so $\mathbb{E}\left(Z_{0}(t+h)-Z_{0}(t)\right)=0$ or equivalently $m_{0}(t)=0$. Using Theorem 3.1 we obtain

$$
\mathbb{E}\left(\boldsymbol{R}_{\alpha}\left(t \wedge c_{1}\right)\right)= \begin{cases}z(t) & \text { if } t<T \\ z(T)+\exp (\alpha T) c_{p}(T)(1-G(T)) & \text { if } t \geq T\end{cases}
$$

with

$$
z(t):=\int_{0}^{t} \exp (-\alpha y) c_{f}(y) r(y)(1-G(y)) d y+\int_{0}^{t} \exp (-\alpha y)(1-G(y)) K(d y)
$$

and so we can determine by Theorem 2.1 and Lemma 2 the behaviour of the function $v_{\alpha}(\cdot)$ (the details are omitted). Observe, if $K(\cdot)$ and/or $c_{p}(\cdot)$ are not 
differentiable, the MCA approach of Berg $[4,5,6]$ cannot be used. Special cases of the above model are discussed by Barlow and Proschan [3], Schaeffer [24], Cleroux and Hanscom [9], Fox [12], Berg and Epstein [7] and Ran and Rosenlund [22]. If we also like to incorporate down times due to repairs we obtain that $c_{i}, i \geq 1$, equals $c_{i}=\left(T \wedge X_{i}\right)+\boldsymbol{L}_{i}$ with $\boldsymbol{L}_{i}$ the length of the $i$ th down time and the random variables $L_{i}, i \geq 1$, independent and identically distributed. In this case the stochastic process $\left\{Z_{0}(t): 0 \leq t<\infty\right\}$ not only counts the costs due to a failure replacement but also incorporates the costs due to down time. In the above model we only allow replacements and we do not allow minimal repairs (Barlow and Proschan [3]) if a component has failed before reaching its critical age $0<T<\infty$. A minimal repair means that the component is restored to its state just before failing or equivalently the failure rate function following a minimal repair is undisturbed (for a mathematical definition see Nakagawa and Kowada [20]). However, if we may choose either to replace a failed component or to repair it minimally we first need to introduce the cost of a minimal repair at age $t$. To model this we introduce a stochastic process $\{\boldsymbol{M}(t): 0 \leq$ $t<\infty$ \} with right continuous sample paths and define

\section{$\boldsymbol{M}(t):=$ costs of a minimal repair if a component fails at age $t$.}

It is realistic to assume that the stochastic process $\{\boldsymbol{M}(t): 0 \leq t<\infty\}$ is increasing and to determine whether a failed component is minimally repaired we also introduce a so-called repair-cost limit function $l:[0, \infty) \rightarrow[0, \infty]$. This function is increasing and right continuous and represents the maximum costs one is willing to pay for a minimal repair of a component of age $t$. This implies that a minimal repair on a failed component of age $t$ is performed if and only if $\boldsymbol{M}(t) \leq l(t)$ and so the probability $p(t)$ that a failed component of age $t$ is minimally repaired is given by $\operatorname{Pr}\{\boldsymbol{M}(t) \leq l(t)\}$. Since the function $l$ is increasing and right continuous and the increasing stochastic process $\{\boldsymbol{M}(t): 0 \leq t<\infty\}$ has right continuous sample paths we obtain that the function $p:[0, \infty) \rightarrow$ $[0,1]$ is right continuous. To determine the random time until the first replacement we consider the stochastic process $\left\{\left(\boldsymbol{Y}_{n}, \boldsymbol{I}_{n}\right): n \geq 1\right\}$ with $\boldsymbol{I}_{n}=0$, resp. 1 , if a minimal repair, resp. a replacement, is carried out at the instance $\boldsymbol{Y}_{n}$, with $\boldsymbol{Y}_{n}$, $n \geq 1$, the instance of the $n$th failure of the first component. Clearly, if $\tau:=$ $\inf \left\{n \geq 1: \boldsymbol{I}_{n}=1\right\}$ then by definition $\boldsymbol{Y}_{n}=\infty$ for every $n \geq \tau+1$ and the random variable $c_{1}$ is represented by $\boldsymbol{c}_{1}=T \wedge \boldsymbol{Y}_{\tau}$. By the definition of minimal repair the point process $\{\boldsymbol{N}(t): 0 \leq t<\infty\}$ associated with $\left\{\boldsymbol{Y}_{n}: n \geq 0\right\}$ (define $Y_{0}:=0$ !) and given by $N(t):=\sup \left\{n \geq 0: Y_{n} \leq t\right\}$ is a nonhomogeneous Poisson process with random intensity function $r(t, \omega)=r(t)$ for every $t \leq \boldsymbol{Y}_{\tau}(\omega)$ and $r(t, \omega)=0$ otherwise. This implies that the failure rate at time $t$ of the distribution of $Y_{\tau}$ equals $(1-p(t)) r(t)$ and hence its distribution function $G$ is given by

$$
1-G(x):=\operatorname{Pr}\left\{\boldsymbol{Y}_{\tau}>x\right\}=\exp \left(-\int_{0}^{x}(1-p(y)) r(y) d y\right)
$$


This yields as already observed by Berg [6] a much easier way to derive the above distribution function than the approach of Block, Borges and Savits [8]. For this model the function $g_{0}$ is the same as for the first model and the right continuous stochastic process $\left\{Z_{0}(t): 0 \leq t<\infty\right\}$ is given by

$$
\begin{aligned}
& Z_{0}(t)= \text { cost in the first cycle up to time } t \text { due to } \\
& \text { minimal repairs and a replacement at failure }
\end{aligned}
$$

As before it is not difficult to verify that $t \rightarrow \mathbb{E}\left(Z_{0}(t)\right)$ is a continuous function. Moreover, by the definition of $c_{1}$ it follows for every $t<T$ that

$$
\begin{aligned}
\mathbb{E}\left(Z_{0}(t+h)-Z_{0}(t) \mid c_{1}>t\right) \\
=\mathbb{E}\left(\left(Z_{0}(t+h)-Z_{0}(t)\right) 1_{\left\{\boldsymbol{Y}_{N(t)+1} \leq t+h, v=N(t)+1\right\}} \mid \boldsymbol{Y}_{\tau}>t\right) \\
\quad+\mathbb{E}\left(\left(Z_{0}(t+h)-Z_{0}(t)\right) 1_{\left\{Y_{N(t)+1} \leq t+h, v>N(t)+1\right\}} \mid \boldsymbol{Y}_{\tau}>t\right)
\end{aligned}
$$

and this yields by the definition of a nonhomogeneous Poisson point process (Ross [23]) that

$$
\begin{aligned}
\lim _{h \downarrow 0} & \frac{\mathbb{E}\left(Z_{0}(t+h)-Z_{0}(t) \mid \boldsymbol{c}_{1}>t\right)}{h} \\
& =(1-p(t)) r(t) c_{f}(t)+r(t) E\left(\boldsymbol{M}(t) 1_{\{\boldsymbol{M}(t) \leq l(t)\}}\right) \\
& =(1-p(t)) r(t) c_{f}(t)+p(t) r(t) E(\boldsymbol{M}(t) \mid \boldsymbol{M}(t) \leq l(t))
\end{aligned}
$$

Moreover, it is also easy to see that $\mathbb{E}\left(Z_{0}(t+h)-Z_{0}(t)\right)=0$ for every $t \geq T$ and $h>0$ and so as before one can work out the expressions for the discounted and average cost criterium (the details are left for the reader). Finally, we like to observe that a special case of the above model is considered by Berg [6] and Block, Borges and Savits [8]. To conclude this section we observe that one can also very easily include in the above analysis a cost function for minimal repairs which not only depends on the age of the component but also on the number of minimal repairs already performed on this component. If one additionally introduces a third option that a minimal repair is unsuccesful with a probability depending on the age and the number of previous minimal repairs and if this happens that one has to replace it by a new component this can still be analyzed using the above approach. This generalization is considered by Makis and Jardine [16]. Also other classes of maintenance models can be analyzed using the above approach. As an example we mention the so-called T-policy of Muth [19] already considered in Section 2 (see also Makis and Jardine [15]). To 
conclude this paper we finally observe that an overview on these other classes of maintenance models is given by Sherif and Smith [25] and Valdez-Flores and Feldman [27].

Acknowledgements: The authors thank one of the referees for his valuable comments which improved the presentation of the paper.

\section{References}

[1] Ash RB (1972) Real analysis and probability. Academic Press, New York

[2] Asmussen S (1987) Applied probability and queues. Wiley, New York

[3] Barlow RE, Proschan F (1967) Mathematical theory of reliability. Wiley, New York

[4] Berg M (1980) A marginal cost analysis for preventive maintenance policies. European Journal of Operational Research 4:136-142

[5] Berg M (1995) The marginal cost analysis and its application to repair and replacement policies. European Journal of Operational Research 82:214-224

[6] Berg M (1996) Economics oriented maintenance analysis and marginal cost approach. In: Özekici S (ed) Reliability and maintenance of complex systems. Springer-Verlag, Berlin

[7] Berg M, Epstein B (1979) A note on a modified block replacement policy for units with increasing marginal running costs. Naval Research Logistics Quarterly 26:157-179

[8] Block HW, Borges WS, Savits TH (1988) A general age replacement model with minimal repair. Naval Research Logistics 35:365-372

[9] Cleroux R, Hanscom M (1974) Age replacement with adjustment and deprecation costs and interest charges. Technometrics 16:235-239

[10] Dekker R, Smeitink E (1991) Opportunity based block replacement. European Journal of Operational Research 53:46-62

[11] Feller W (1971) An Introduction to probability theory and its applications, volume 2. Wiley, New York

[12] Fox B (1966) Age replacement with discounting. Operations Research 14:535-537

[13] Frenk JBG, Dekker R, Kleijn MJ (1996) A unified treatment of single component replacement models. Technical Report 9602/A, Econometric Institute, Erasmus University Rotterdam,

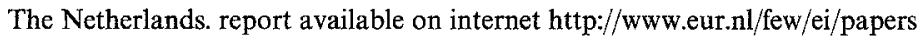

[14] Loeve M (1977) Probability theory I. Springer-Verlag, New York

[15] Makis V, Jardine AKS (1991) Optimal replacement of a system with imperfect repair. Microelectron. Reliability $31: 381-388$

[16] Makis V, Jardine AKS (1992) Optimal replacement policy for a general model with imperfect repair. Journal of the Operational Research Society 43:111-120

[17] McCall JJ (1965) Maintenance policies for stochastically failing equipment: A survey. Management Science 11:493-524

[18] Mikolás M (1994) Real functions, Abstract spaces and orthogonal series. Akademia Kiadó, Budapest

[19] Muth EJ (1977) An optimal decision rule for repair vs. replacement. IEEE Transactions on Reliability 26:179-181

[20] Nakagawa T, Kowada M (1988) Analysis of a system with minimal repair and its application to replacement policy. European Journal of Operational Research 12:176-182

[21] Pierskalla WP, Voelker JA (1979) A survey of maintenance models: the control and surveillance of deteriorating systems. Naval Research Logistics Quarterly 23:353-388 
[22] Ran A, Rosenlund SI (1976) Age replacement with discounting for a continuous maintenance cost model. Technometrics 18:459-465

[23] Ross SM (1970) Applied probability models with optimization applications. Holden-Day, San Francisco

[24] Schaeffer RL (1971) Optimum age replacement with an increasing cost factor. Technometrics 13:139-144

[25] Sherif YS, Smith ML (1981) Optimal maintenance models for systems subject to failure - a review. Naval Research Logistics Quarterly 28:47-74

[26] Sivazlian BD (1973) On the discounted replacement problem with arbitrary repair time distribution. Management Science 19:1301-1309

[27] Valdez-Flores C, Feldman RM (1989) A survey of preventive maintenance for stochastically deteriorating single component systems. Naval Research Logistics Quarterly 36:419-446

[28] Van Weeren S (1995) Single-component maintenance and fractional programming. Master Thesis, Econometric Institute, Erasmus University Rotterdam, The Netherlands

[29] Wolff RW (1989) Stochastic modelling and the theory of queues. Prentice Hall, London

[30] Zijlstra M (1979) Regenerative replacement policies with general cost structures. ISAResearch-SP note 92 (second revised edition), N. V. Philips, Eindhoven

[31] Zijlstra M (1981) Renewal replacement policies for one unit systems. European Journal of Operational Research 8:289-293 